\title{
Elastase and Cathepsin G from Primed Leukocytes Cleave Vascular Endothelial Cadherin in Hemodialysis Patients
}

\author{
Meital Cohen-Mazor, ${ }^{1,2}$ Rafi Mazor, ${ }^{1,2}$ Batya Kristal, ${ }^{3,4}$ and Shifra Sela ${ }^{2,4}$ \\ ${ }^{1}$ Bruce Rappaport School of Medicine, Technion, 31096 Haifa, Israel \\ ${ }^{2}$ Eliachar Research Laboratory, Western Galilee Hospital, 22100 Naharyia, Israel \\ ${ }^{3}$ Nephrology Unit, Western Galilee Hospital, 22100 Nahariya, Israel \\ ${ }^{4}$ Faculty of Medicine in the Galilee, Bar Ilan University, 13100 Safed, Israel
}

Correspondence should be addressed to Meital Cohen-Mazor; mazmeital@gmail.com

Received 3 February 2014; Accepted 31 March 2014; Published 4 May 2014

Academic Editor: Beatrice Charreau

Copyright (c) 2014 Meital Cohen-Mazor et al. This is an open access article distributed under the Creative Commons Attribution License, which permits unrestricted use, distribution, and reproduction in any medium, provided the original work is properly cited.

\begin{abstract}
Aims. To test the hypothesis that primed PMNLs in blood of chronic kidney disease patients release the active form of elastase and cathepsin G causing degradation of vital proteins and promote tissue damage. Methods. RT-PCR, immunocytochemical staining, immunoblotting, and FACS analyses were used to study these enzymes in hemodialysis patients (HD) versus healthy normal controls (NC). Using PMNLs and endothelial cells cocultivation system we measure the effect of HD PMNLs on the endothelial VE-cadherin, an essential protein for maintaining endothelial integrity. Results. Levels of elastase and cathepsin $\mathrm{G}$ were reduced in PMNLs of HD patients, while mRNA enzymes levels were not different. Elevated levels of the active form of these enzymes were found in blood of HD patients compared to NC.HD plasma had higher levels of soluble VE-cadherin present in three molecular forms: whole $140 \mathrm{kDa}$ molecule and two fragments of 100 and $40 \mathrm{kDa}$. Cocultivation studies showed that primed PMNLs cleave the endothelial cadherin, resulting in a $100 \mathrm{kDa}$ fragment. Conclusions. Elastase and cathepsin G are elevated in the plasma of HD patients, originating from primed PMNLs. In these patients, chronic elevation of these enzymes contributes to cleavage of VEcadherin and possible disruption of endothelial integrity.
\end{abstract}

\section{Introduction}

Elastase and cathepsin G are serine proteinases contained in the azurophilic granules of peripheral polymorphonuclear leukocytes (PMNLs) and degrade proteins of phagocytosed microorganisms $[1,2]$. Stored after synthesis in their active form, these proteolytic enzymes have potential deleterious effects. When released, they act on substrates comprising the connective tissue of the lung, blood vessels, joints, and other organs, causing tissue destruction [3-9]. Circulating proteinase inhibitors, such as serum proteins $\alpha 1$-antitrypsin $(\alpha 1-\mathrm{AT})$ and $\alpha 1$-antichymotrypsin $(\alpha 1-\mathrm{ACT})$, neutralize the activity of these proteinases in plasma [10, 11]. The clinical significance of elastase and cathepsin $\mathrm{G}$ is most evident under conditions in which an imbalance develops between these enzymes and their inhibitors, enabling the circulating active enzymes to induce vascular injury.
PMNL serine proteinases have been implicated in the pathogenesis of diseases associated with PMNL priming such as rheumatoid arthritis [12, 13]. PMNL priming is also associated with pathological disorders associated with atherosclerosis and cardiovascular diseases such as hypertension, diabetes, and end stage renal disease treated with chronic hemodialysis (HD) [14-16]. Studies have shown that purified elastase and cathepsin $\mathrm{G}$ can cleave the extracellular part of vascular endothelial (VE) cadherin, an essential protein for maintaining vascular endothelial integrity $[17,18]$, and that elastase and cathepsin $\mathrm{G}$ bound to their inhibitor are released during hemodialysis sessions [19].

In this study, we sought out to test the hypothesis that even before dialysis HD PMNLs are primed, thus contributing to elevated levels of elastase and cathepsin $G$ in the plasma. We have also evaluated cleavage of VE-cadherin mediated by these proteinases found in HD plasma. 
Our findings consist of elevated levels of these proteins in HD plasma. Plasma of HD had higher levels of VE-cadherin fragments, the result of this molecule cleavage by active elastase and cathepsin G. HD PMNLs as well as purified enzymes cleaved VE-cadherin from cultured endothelial cells. Taken together, this study provides a novel mechanism by which active elastase and cathepsin G originating from primed PMNLs of HD patients can initiate endothelial dysfunction.

\section{Materials and Methods}

2.1. Patients and Blood Samples. Ten hemodialysis patients and healthy control subjects were enrolled in this study (Table 1). Blood for the determination of biochemical and hematological parameters and for the isolation of PMNLs was drawn into citrate tubes, and in $\mathrm{HD}$ patients from the arterial line immediately before a dialysis session. All patients underwent hemodialysis three times a week; each dialysis treatment lasted 4 hours and was carried out with low flux polysulfone membranes (F8, Fresenius Medical Care, Bad Homburg, Germany). The water for dialysis met the standards of the Association for the Advancement of Medical Instrumentation (AAMI). Patients with evidence of acute or chronic infection, malignancy, or who had received a blood transfusion within three months prior to blood sampling were excluded. All participants signed an informed consent for blood sampling, and the study was approved by the Institutional Committee in accordance with the Helsinki declaration.

2.2. PMNL Isolation and Analysis. PMNLs were isolated as described previously [15]. Isolated PMNLs (>98\% pure, approximately $10^{7}$ cells per isolation) were resuspended and counted in phosphate buffered saline (PBS, Beit Haemek, Israel) containing $0.1 \%$ glucose. PMNL priming was assessed by the rate of superoxide release [14], and by the surface levels of CD11b, as described previously [20]. The rate of superoxide release was determined after cell stimulation with $0.32 \times 10^{-7} \mathrm{M}$ phorbol 12-myristate 13-acetate (PMA, Sigma, St. Louis, MO). The assay is based upon superoxide dismutase (SOD) inhibitable reduction of $80 \mu \mathrm{M}$ cytochrome C (Sigma, St. Louis, MO) to its ferrous form. The change in optical density was monitored at $549 \mathrm{~nm}$, as described previously [14]. Expression of CD11b on PMNLs in whole blood was determined using the FC500 flow cytometer (BeckmanCoulter). Fifty $\mu \mathrm{L}$ of blood was incubated for $10 \mathrm{~min}$ with anti CD11b-PE conjugated monoclonal antibody (IQ Products, The Netherlands), followed by RBC lysis (Q-prep method; Coulter Corporation, Hialeah, FL, USA). To enable gating on the PMNL population, anti CD16, conjugated to PC5 monoclonal antibody (Immunotech, Marseille, France), was used. Surface levels of CD11b on PMNLs are expressed as mean fluorescence intensity (MFI), after subtracting the nonspecific background.

2.3. RNA Isolation and RT-PCR of Elastase and Cathepsin $G$. RNA was isolated using Tri Reagent according to the manufacturer's (Sigma) instructions. Complementary DNA
TABLE 1: Demographics of HD patients and NC subjects used in this study.

\begin{tabular}{lcc}
\hline & $\begin{array}{c}\text { Hemodialysis } \\
\text { patients }(n=10)\end{array}$ & $\begin{array}{c}\text { Normal controls } \\
(n=10)\end{array}$ \\
\hline Age (years) & $54 \pm 7$ & $47 \pm 5$ \\
Male/Female & $5 / 5$ & $5 / 5$ \\
$\begin{array}{l}\text { Diabetes } \\
\text { Time on hemodialysis } \\
\text { (months) }\end{array}$ & $38 \pm 6$ & - \\
\hline
\end{tabular}

was synthesized according to Krug and Berger [21], using an OmniGene thermal cycler (Hybaid, UK). The resulting cDNAs were amplified by PCR using $2 \mu \mathrm{L}$ aliquots of RT reaction incubated with $0.2 \mathrm{mM}$ dNTPs, $2 \mu \mathrm{L}$ of Taq DNA polymerase buffer, $0.6 \mathrm{U}$ of TaqDNA polymerase (BioLine, UK), and 10 pmoles of the specific primers. The oligonucleotide sequence for elastase, according to Hirata et al. [22], is sense $\left(5^{\prime}\right.$-AGTGCCTGGCCATGGGCTGG- $\left.3^{\prime}\right)$ and antisense $\left(5^{\prime}\right.$ CACCGGGGCAAAGGCATCGG- $3^{\prime}$ ) with a final PCR product of $257 \mathrm{bp}$. The PCR conditions were initial denaturation at $94^{\circ} \mathrm{C}$ for 5 minutes, 30 PCR cycles of denaturation at $94^{\circ} \mathrm{C}$ for 1 minute, annealing at $55^{\circ} \mathrm{C}$ for 2 minutes, and extension at $72^{\circ} \mathrm{C}$ for 3 minutes. The oligonucleotide sequence for cathepsin $\mathrm{G}$, according to Hirata et al. [22], is sense $\left(5^{\prime}\right.$-TGAGAGTGCAGAGGGATAGG-3') and antisense $\left(5^{\prime}\right.$ CAGGAAACTTGAGACCCTGC- ${ }^{\prime}$ ) with a final PCR product of $216 \mathrm{bp}$. The PCR conditions were identical to those described above for elastase. Aliquots $(15 \mu \mathrm{L})$ of the amplified cDNA of elastase and cathepsin $\mathrm{G}$ were separated on $1 \%$ agarose gel electrophoresis, visualized by ethidium bromide staining, and compared to a housekeeping gene, $\beta$-actin. The $200 \mathrm{bp}$ product of actin was amplified using the following primers: sense ( $5^{\prime}$-CCTTCCTGGGCATGGAGTCCTG-3'), and antisense $\left(5^{\prime}\right.$-GAGCAATGATCTTGATCTTC- $\left.3^{\prime}\right)$. The densities of the enzyme PCR products were calculated in each gel relative to the actin product.

\subsection{Detection and Quantificationof Elastase and Cathepsin $G$ in PMNLs}

(1) Intracellular Levels of Elastase and Cathepsin G in PMNLs. The levels of intracellular elastase and cathepsin G were measured in whole blood by flow cytometry using polyclonal rabbit anti-human elastase and anti-human cathepsin G antibodies (United States Biological, Massachusetts, USA), followed by a secondary FITC-conjugated fluorescent antirabbit antibody (Chemicon International, CA). PMNLs were permeabilized with the FIX \& PERM cell permeabilization kit. Anti CD16- PC5 (Immunotech, Marseille, France) was used for gating on PMNLs. Total rabbit IgG antibodies (United States Biological, Massachusetts, USA), followed by a secondary FITC-conjugated fluorescent anti-rabbit antibody, served as nonspecific controls of the fluorochrome. The results are presented as MFI per cell after subtracting the nonspecific background. 
(2) Localization of Elastase and Cathepsin G in PMNLs. Human PMNLs were stained by indirect immunohistochemical staining. Whole blood slides were prepared by fixation and permeabilization with methanol $\left(-20^{\circ} \mathrm{C}, 3\right.$ minutes). The slides were stained with specific anti-elastase and anti-cathepsin G antibodies (United States Biological, Massachusetts, USA). The Histofine Simple Stain kit (Nichirei Corporation, Japan) was used for immunohistochemical staining of PMNLs according to the manufacturer's instructions.

2.5. Detection and Quantification of Elastase and Cathepsin $G$ in Plasma. Plasma of NC subjects and HD patients was depleted of albumin and immunoglobulins by ProteoPrep Blue Albumin Depletion Kit (PROT-BA KIT) (SigmaAldrich, USA). Following the addition of Laemli loading buffer containing 3\% $\beta$-mercaptoethanol, the plasma was boiled at $100^{\circ} \mathrm{C}$ for 5 minutes. Samples were loaded onto $15 \%$ polyacrylamide SDS gels for separation of proteins and then transferred to nitrocellulose filters by semidry transfer (Biometra, Germany). The filters were blocked by $1 \%$ fat milk for 1 hour at room temperature and then incubated at room temperature, first with rabbit anti-human elastase/rabbit anti-human cathepsin $G$ antibodies (Fitzgerald Industries International, USA) (diluted 1:1,000) for 2 hours, followed by incubation with goat anti rabbit-HRP conjugate (diluted $1: 25,000)$ for an additional hour. The amount of the enzyme in each sample was calculated according to a positive control loaded on the same gel, active elastase (Sigma-Aldrich-cat. number E-8140)/active cathepsin G (Fitzgerald Industries International, USA).

The enzyme signal was detected on X-ray films using the chemiluminescence reagents of the EZ-ECL kit. The densities of the enzyme bands were determined by the BioCapt and Bio-Profile (Bio-1D) software.

2.6. Endothelial Cell Culture. Human umbilical vein endothelial cells (HUVEC) were cultured as described by Jaffe et al. [23], with minor modifications according to Lanir et al. [24]. Endothelial cell specificity (>95\%) was confirmed by flow cytometry of cells stained with anti-human CD-34 PE-conjugated antibody (Becton Dickinson, USA) after cell detachment by trypsin/EDTA (Biological Industries, Beit Haemek, Israel).

2.7. Cocultivation of HUVEC with PMNLs. HUVEC were seeded in growth medium at a density of $1 \times 10^{5}$ cells in six-well plates (Nalge Nunc International, USA) for 2 days under subconfluent conditions. Experiments were performed always between passages 2 to 4 . In all experiments, prior to the addition of PMNLs, HUVEC were deprived of culture medium and kept in phosphate buffered saline (PBS) for $90 \mathrm{~min}$ at $37^{\circ} \mathrm{C}$ [25]. To prevent the direct contact of PMNLs with HUVEC, $0.45 \mu \mathrm{m}$ pore size cell culture inserts $(25 \mathrm{~mm}$ tissue culture inserts, Nalge Nunc International) were placed into each well on top of HUVEC in direct contact with the medium, $1 \mathrm{~mL}$ of PBS [26]. PMNLs $\left(10^{6}\right)$ from NC or HD were seeded on these inserts and coincubated for 15 minutes at $37^{\circ} \mathrm{C}$ with HUVEC, as previously described [25].

\subsection{Assessment of VE-Cadherin on HUVEC}

(1) By Flow Cytometry. HUVEC, following 15 minute of cocultivation with PMNLs, were rinsed with PBS, scraped, centrifuged, resuspended, and incubated for 10 minutes with mouse anti VE-cadherin monoclonal antibody (United States Biological, MA, USA), followed by incubation with PE conjugated anti-mouse antibody (IQ products, The Netherlands). Irrelevant IgG-PE served as a nonspecific control of the fluorochrome (IQ products, The Netherlands).

(2) By Immunohistochemical Staining. HUVEC were grown on a chamber, a procedure that does not require cell transfer prior to visualization/staining, and cocultivated with PMNLs as previously described [25]. After cocultivation, the growth chamber was removed, and cells were fixed on the slides in $95 \%$ ethanol and stained with mouse anti VE-cadherin monoclonal antibody (United States Biological, MA, USA).

\subsection{Evaluation of Soluble VE-Cadherin Fragments}

(1) In Plasma. Soluble VE-cadherin was measured using western blot analysis. The plasma of NC subjects and HD patients was depleted of albumin and immunoglobulins. Following the addition of Laemli loading buffer containing $3 \% \beta$-mercaptoethanol, the plasma was boiled at $100^{\circ} \mathrm{C}$ for 5 minutes. Samples were loaded onto $6 \%$ polyacrylamide SDS gels as described above. The whole $140 \mathrm{kDa}$ molecule and the $100 \mathrm{kDa}$ fragment were detected using mouse anti-human VE-cadherin (Cell Signaling Technology, MA, USA), while the $40 \mathrm{kDa}$ fragment was detected using mouse anti-human VE-cadherin (United States Biological, Massachusetts, USA), which is not able to recognize the $140 \mathrm{kDa}$ and the $100 \mathrm{kDa}$ molecules. Thus, due to the different antibodies used, plasma sampled was loaded onto two gels, one stained for the evaluation of $100+140 \mathrm{kDa}$ forms and one for the $40 \mathrm{kDa}$ form. The levels of both whole and cleaved forms of VEcadherin were assessed in HD plasma and compared with those of NC plasma. To calculate the levels of the enzymes, the bands were all compared to the same plasma sample run on all gels serving as a normalizing control.

(2) In the Cocultivation Media. The media of four cocultivation experiments were pooled in order to get detectable amount of these proteins. These media samples were treated with Vivaspin concentrators (Sartorius AG, Germany) for improved recovery of the low-concentration protein samples. Following the addition of Laemli loading buffer containing $3 \% \beta$-mercaptoethanol, the plasma was boiled at $100^{\circ} \mathrm{C}$ for 5 minutes. Samples were loaded onto $6 \%$ polyacrylamide SDS gels as described above using mouse anti-human VEcadherin (Cell Signaling Technology, MA, USA).

2.10. Statistical Analysis. Data is expressed as mean \pm SD. In the boxes and whiskers presentations, the horizontal line 
in the middle shows the median (50th percentile), the top and bottom of the box show the 75th and 25th percentiles, respectively, and the whiskers show the maximum and the minimum values. The nonparametric Mann-Whitney test was used for comparing two independent groups. The twopaired Wilcoxon Signed Ranks test was used for comparing two dependent groups. Statistical significance was considered at $P<0.05$.

\section{Results}

3.1. PMNLs Priming in HD Patients. We reported previously that PMNLs from HD patients are primed [16]. To confirm these results we isolated PMNLs and measured the rate of superoxide release and the levels of surface CD11b, indices of PMNL priming [20]. The rate of superoxide release following PMA stimulation was higher in PMNLs isolated from HD patients than in those from NC $(38.5 \pm 3.9$ versus $24.7 \pm 5$ nmoles $/ 10^{6}$ cells $/ 10 \mathrm{~min}$, resp., $\left.P<0.05\right)$. The membrane levels of CD11b were higher in PMNLs isolated from HD patients than in PMNLs from NC $(49.9 \pm 7.8$ versus $32 \pm 4$ MFI resp., $P<0.05)$.

3.2. Elastase and Cathepsin G mRNA in PMNLs. Elastase and cathepsin $\mathrm{G}$ mRNA levels were not significantly different in NC versus HD PMNLs: $0.39 \pm 0.14$ versus $0.33 \pm 0.22$ relative density units, respectively, for elastase; $P=$ ns; and $0.74 \pm$ 0.1 versus $0.86 \pm 0.47$ relative density units, respectively, for cathepsin $\mathrm{G} ; P=\mathrm{ns}$.

3.3. PMNL Intracellular Protein Levels of Elastase and Cathep$\sin G$. Flow cytometry measurements of elastase and cathepsin G in PMNLs measured in whole blood (Figures 1(a)-1(e)) showed higher levels of these enzymes in NC PMNLs than in HD PMNLs (average of 22.9 \pm 2.7 versus $12.3 \pm 1.7 \mathrm{MFI}$, resp., for elastase; $P<0.05$ and $35 \pm 5.2$ versus $12.8 \pm 1.4 \mathrm{MFI}$, resp., for cathepsin $\mathrm{G} ; P<0.05)$.

A significant negative correlation was found between the fluorescent intensity of intracellular elastase and cathepsin G and membrane CD11b expression on PMNLs, $(r=-0.43$ and $r=-0.51$, resp.; $P<0.05$ ). This negative correlation indicates that the higher the priming the lower the amount of the intracellular enzymes.

3.4. Immunohistochemical Staining of Elastase and Cathepsin $G$ in PMNLs. The intracellular levels and locations of elastase and cathepsin G were also evaluated by immunohistochemical staining of PMNLs in smears of whole blood, as represented in Figure 2. Examination of the cells under a light microscope revealed that elastase and cathepsin $\mathrm{G}$ were abundant and distributed in PMNLs of NC, while sparse in HD PMNLs, and even missing in some patients.

3.5. Plasma Levels of Elastase and Cathepsin G. Plasma of $\mathrm{NC}$ and HD was depleted of albumin and immunoglobulins in order to enrich it with elastase and cathepsin G. After depletion, plasma proteins were separated on SDS-PAGE followed by western blot analysis (Figure 3). HD plasma contained higher levels of free elastase and cathepsin G $(30 \mathrm{KDa})$ than $\mathrm{NC}$ plasma (average of $1.3 \pm 0.14$ versus $0.76 \pm 0.08$ relative density units, resp., for plasma elastase; $P<0.05$ and $0.52 \pm 0.04$ versus $0.34 \pm 0.05$ relative density units, resp., for plasma cathepsin G; $P<0.05$ ) (Figures 3(b) and $3(\mathrm{c}))$. However, no significant differences in the levels of the higher molecular weight complexes (40,50, and $70 \mathrm{Da})$ were found between NC and HD. A significant negative correlation was found between the amounts of the plasma levels of elastase and cathepsin $\mathrm{G}(30 \mathrm{KDa})$ and their levels in PMNLs (Figures 3(d) and 3(e)) ( $r=-0.5$ and $r=-0.6$, resp.; $P<0.05)$.

3.6. Plasma Levels of Soluble VE-Cadherin Fragments. A significant difference in the levels of soluble VE-cadherin between NC and HD plasma was found: HD plasma contains higher levels of soluble VE-cadherin, both the whole molecule, $140 \mathrm{KDa}(1.46 \pm 0.07$ versus $1.25 \pm 0.06$ relative density units; $P<0.05$ ), and the cleaved form, $100 \mathrm{KDa}$ $(0.47 \pm 0.06$ versus $0.3 \pm 0.04$ relative density units; $P<0.05)$ (Figures 4(a) and 4(b)). Using anti VE-cadherin monoclonal antibody, we were able to detect the $40 \mathrm{kDa}$ fragment of VEcadherin in plasma. Again, HD plasma contains higher levels of $40 \mathrm{kDa}$ VE-cadherin $(0.5 \pm 0.07$ versus $0.27 \pm 0.06$ relative density units; $P<0.05$ ) (Figures $4(\mathrm{a})$ and $4(\mathrm{~b})$ ).

In addition, significant positive correlations were found between plasma active elastase and cathepsin $G$ and the $100 \mathrm{KDa}$ soluble VE-cadherin (Figures 5(a) and 5(b)) $(r=$ 0.51 and $r=0.53$ for elastase and cathepsin G, resp.; $P<0.05)$, suggesting that these proteins are involved in the degradation of VE-cadherin. No significant correlation was found between plasma active elastase and cathepsin $G$ and the 140 and $40 \mathrm{KDa}$ VE-cadherin forms.

3.7. HUVEC Membrane $40 \mathrm{kDa}$ form of VE-Cadherin after Exposure to PMNLs. Control HUVEC and HUVEC exposed to NC PMNLs show light staining which indicates that the anti VE-cadherin did not bind to the VE-cadherin (Figure 6). However, when HUVEC were exposed to HD PMNLs, the staining was much intense, indicating strong binding of the antibody to VE-cadherin (Figure 6). When HUVEC were exposed only to proteases $(0.02 \mathrm{U} / \mathrm{mL}$ elastase and $0.02 \mathrm{U} / \mathrm{mL}$ cathepsin G [12]), VE-cadherin staining was comparable to that after exposure to HD PMNLs. HUVEC membrane VE-cadherin was also determined by flow cytometry after exposure to HD and NC PMNLs. The level of VE-cadherin on unexposed HUVEC was 7.03 $\pm 3.84 \mathrm{MFI}$. Cocultivation of HUVEC with NC PMNLs resulted in a significant increase in VE-cadherin staining compared to untreated HUVEC $(9.85 \pm$ 5.77; $P<0.05)$. Cocultivation of HUVEC with HD PMNLs resulted in a greater increase in VE-cadherin staining (13.4 \pm 7.57; $P<0.05)$.

3.8. Cocultivation Media Levels of Soluble $100 \mathrm{kDa}$ Cleaved form of VE-Cadherin. We could not detect any soluble VEcadherin in the control HUVEC which was not exposed to PMNLs. On the other hand, there was a detectable amount of soluble $100 \mathrm{kDa} \mathrm{VE}$-cadherin in the cocultivation media 


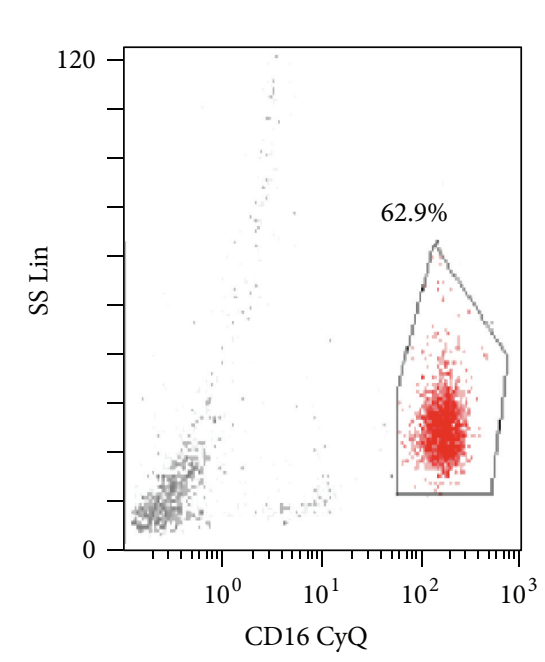

(a)

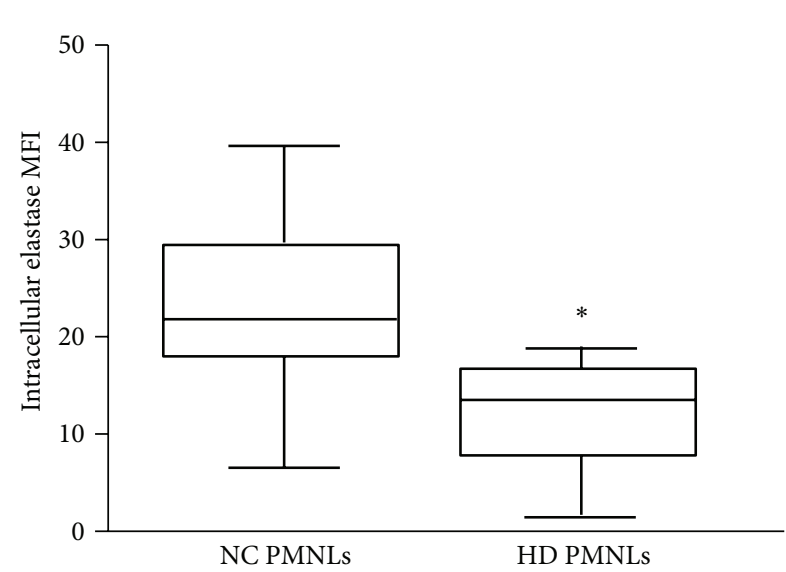

(d)

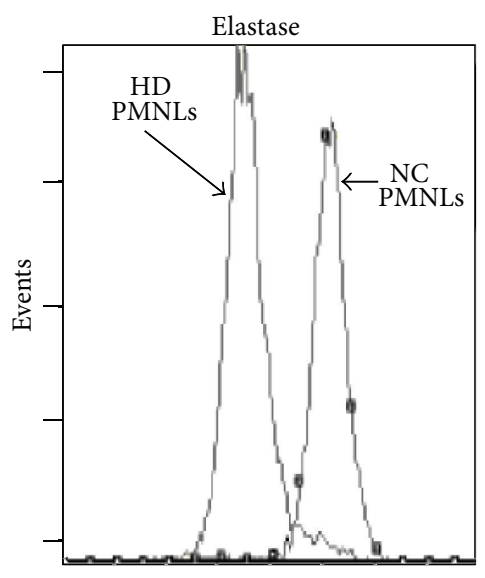

Fluorescence intensity-FITC

(b)

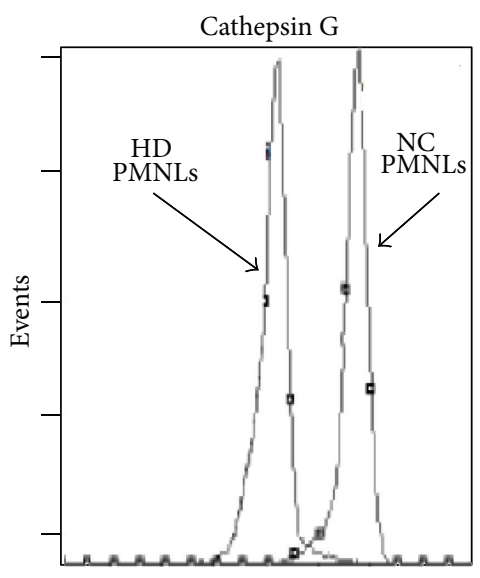

Fluorescence intensity-FITC

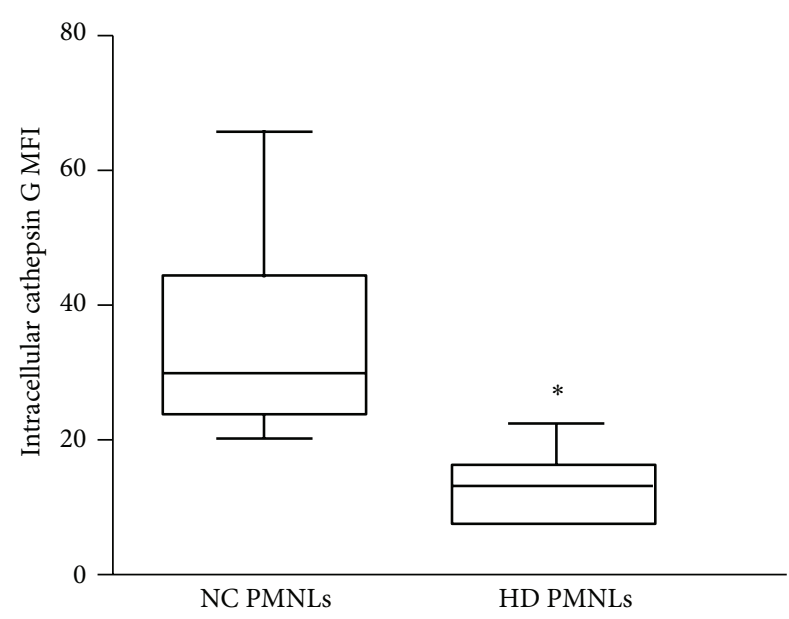

(e)

Figure 1: Intracellular levels of elastase and cathepsin G in PMNLs measured in whole blood. (a) Representative histogram of flow cytometry showing gating on the PMNL population which is CD16 positive cells. (b, c) Representative histogram of flow cytometry showing intracellular elastase and cathepsin G intensity in HD and NC PMNLs, respectively. (d, e) PMNL intracellular elastase and cathepsin G from NC subjects and HD patients detected by flow cytometry $(n=10) .{ }^{*} P<0.05$ HD versus NC.

when HUVEC were exposed to PMNLs (Figure 7). When HUVEC were exposed to HD PMNLs we detected 3 times more soluble VE-cadherin versus when exposed to NC PMNLs (Figure 7). We could not detect the 140 and $40 \mathrm{kDa}$ forms of VE-cadherin in the media.

\section{Discussion}

The results of the present study demonstrate that the intracellular levels of elastase and cathepsin G in primed peripheral PMNLs of HD patients, before starting hemodialysis session, are significantly lower compared to healthy controls although their mRNA levels are similar. Lower intracellular levels of these proteases are associated with a significant increase in their plasma concentration, especially of the active form. This suggests that PMNL priming, common to HD patients, causes an increased release of these enzymes, resulting in their higher plasma levels in HD. Plasma from HD patients also contains higher levels of soluble VE-cadherin, in three molecular forms: $140 \mathrm{kDa}$, the whole molecule and two molecular fragments of this protein, 100 and $40 \mathrm{kDa}$.

We investigated the degree of PMNL priming of $\mathrm{HD}$ patients and the possible correlation between PMNL priming and the release of elastase and cathepsin G. First we supported our previous studies showing that PMNLs in HD patients are primed by using two different markers: elevated levels of CD11b and higher rate of superoxide release. Next, using immunocytochemical staining, immunoblotting, and FACS analyses we demonstrated that the staining intensities of the intracellular elastase and cathepsin $G$ are reduced in the primed PMNLs of HD patients versus NC. A significant negative correlation was found between the intensity of intracellular elastase and cathepsin $G$ and the degree of PMNL priming, as assessed by membrane CD11b expression, suggesting that the release of these proteolytic enzymes is greater when PMNLs are primed. Moreover, our findings 
Elastase

NC

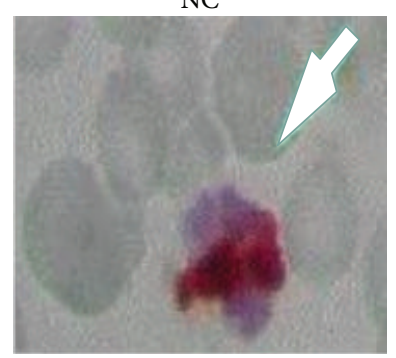

(a)

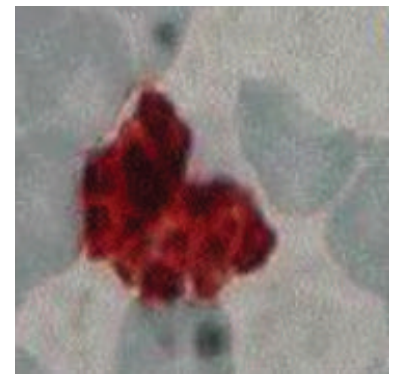

(b)
HD

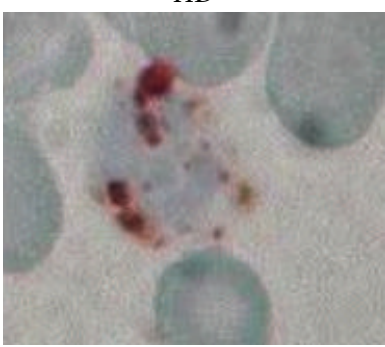

(c)

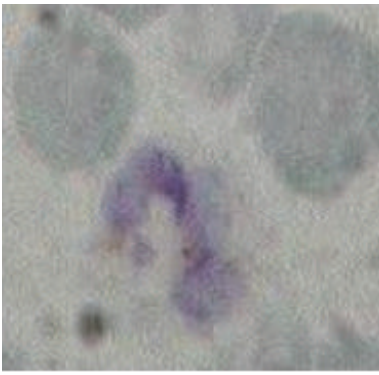

(d)
Cathepsin $G$

NC

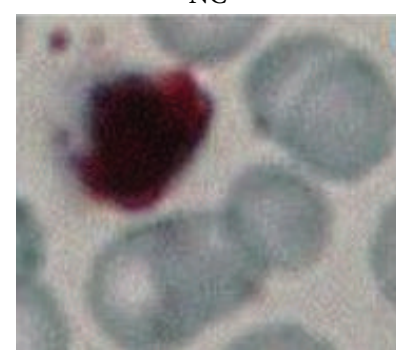

(e)

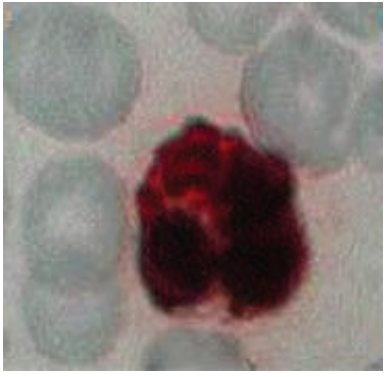

(f)
HD

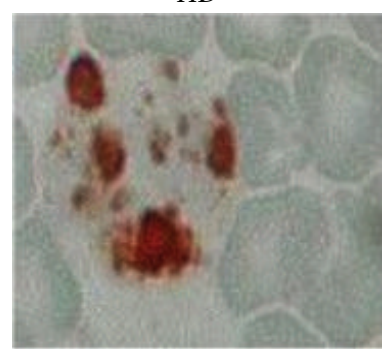

(g)

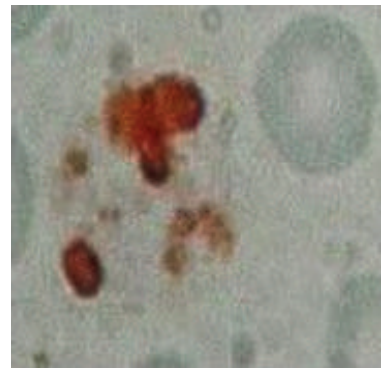

(h)

FIGURE 2: Localization of elastase and cathepsin G in PMNLs in whole blood smears. Indirect immunostaining of elastase (a-d) and cathepsin $\mathrm{G}(\mathrm{e}-\mathrm{h})$ in blood smears of two NC subjects and two HD patients (light microscopy, magnification $\times 100)$.

refute the possibility that lower synthesis of these enzymes in the HD PMNLs caused their reduced intracellular levels, since the transcription levels of these enzymes were comparable in HD and healthy controls. This is in accordance with previous studies demonstrating that azurophilic granule proteins, such as these serine proteinases, are synthesized only at the promyelocyte and metamyelocyte stages, and remain stored in granules throughout terminal granulocytic differentiation $[27,28]$.

Since the intracellular protein levels of these enzymes were lower in $\mathrm{HD}$, one would expect to find these enzymes in the surrounding milieu, the blood. As expected, significantly elevated levels of both proteolytic enzymes were found in blood of HD patients compared to NC.

The continuous release of these enzymes from PMNLs was already demonstrated during hemodialysis sessions [19], but to the best of our knowledge, a comparison with healthy subjects, especially as to the amounts of the active, noninhibitor bound enzymes, was not reported. Although the plasma levels of the higher molecular weights complexes of the enzymes $(40,50$, and $70 \mathrm{kDa})$ were similar between plasma of $\mathrm{HD}$ and $\mathrm{NC}$, the levels of the $30 \mathrm{kDa}$ inhibitor unbound enzymes were significantly higher in HD plasma. Enzyme-inhibitor complexes are formed when elastase or cathepsin $\mathrm{G}$ binds to inhibitory proteins of the acute phase, such as serum proteins $\alpha 1$-antitrypsin $(\alpha 1-\mathrm{AT})$ and $\alpha 1$ antichymotrypsin ( $\alpha 1$-ACT) [10, 11]. The significant negative correlation between intracellular elastase and cathepsin $\mathrm{G}$ and the degree of PMNL priming and plasma levels of these enzymes further supports the enhanced degranulation of these primed PMNLs resulting in the release of the two proteolytic enzymes. This uncontrolled degranulation of primed PMNLs to the blood is potentially destructive in two ways: (1) reduced intracellular levels of these enzymes within the PMNLs decrease their capacity to neutralize and kill pathogens; (2) the continuous interactions of circulating HD PMNL with the blood vessel endothelial monolayer expose the vascular wall to chronic injury by these active circulating granular proteinases.

In the "response-to-injury" hypothesis suggested by Ross, atherosclerosis begins as a response to chronic minimal injury to the endothelium [29]. This injury leads to an array of endothelial cell responses, such as disruption of endothelial integrity, which will in the long run result in atherosclerosis [30]. Adherence junction (AJs) proteins, such as VE-cadherin, maintain endothelial integrity. Hermant at al. have shown that cleavage of VE-cadherin occurs following adhesion of fMLP-primed neutrophils to endothelial cell monolayer resulting in a soluble fragment of molecular mass of $38 \mathrm{KDa}$. Using specific inhibitors of neutrophil proteases, these researchers were able to identify elastase and cathepsin $G$ as the major proteases involved in the cleavage of VE-cadherin. In addition, they demonstrated that purified elastase and cathepsin G are able to increase endothelial monolayer permeability in vivo [17]. Soeki et al. have shown that enhanced secretion of VE-cadherin from the arteries is associated with coronary atherosclerosis [31]. We imply that the elevated plasma levels of elastase and cathepsin $\mathrm{G}$ in $\mathrm{HD}$ patients, especially the active forms, cleave AJs proteins, such as VE-cadherin in the endothelium, an action associated with early steps in the atherosclerotic process. 


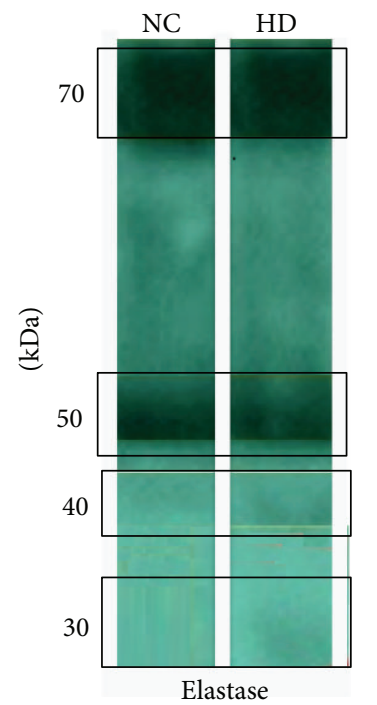

(a)

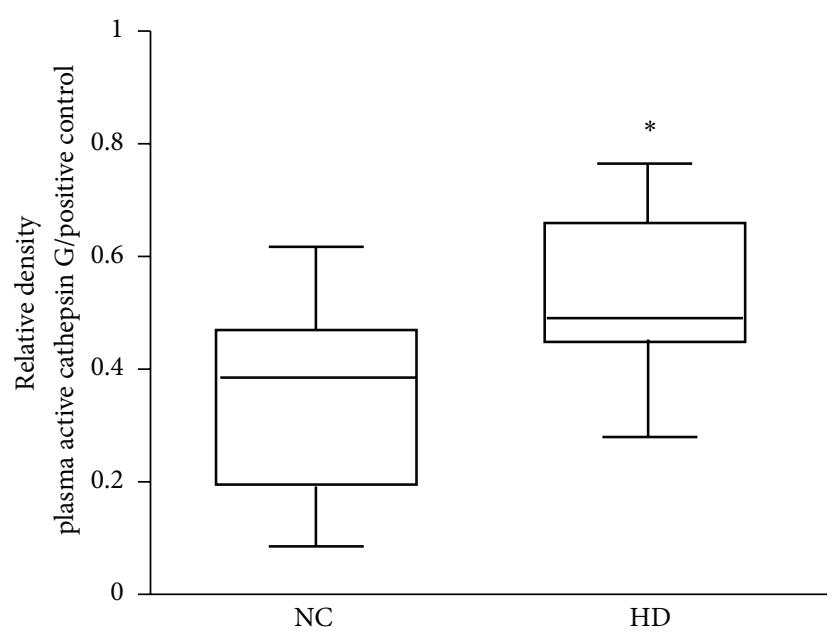

(c)

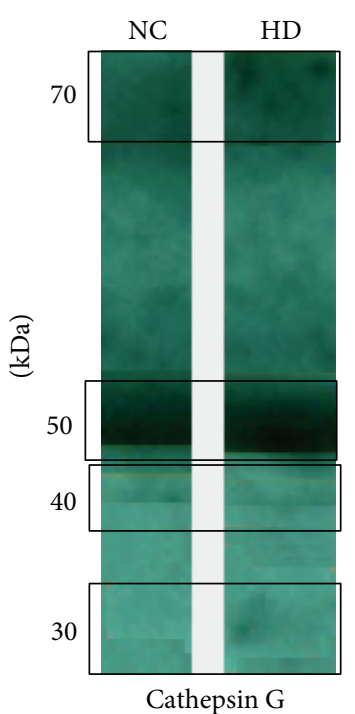

Cathepsin G

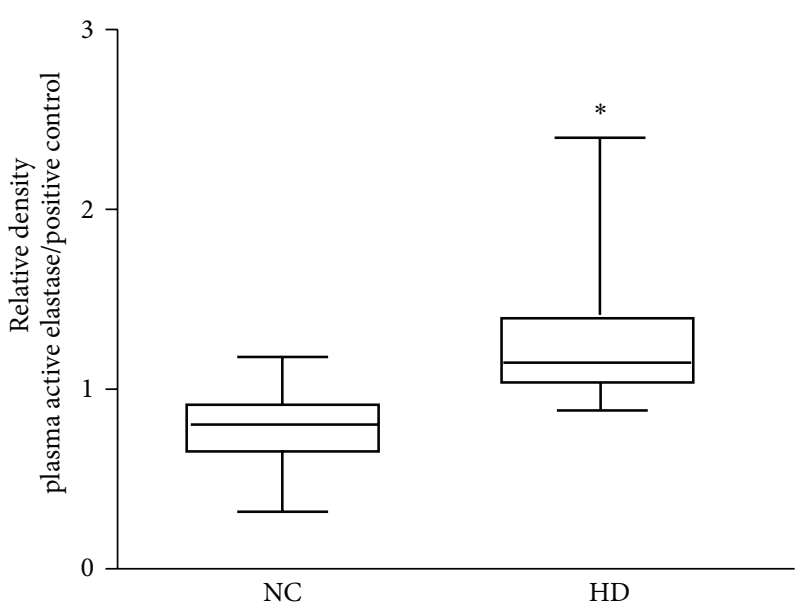

(b)

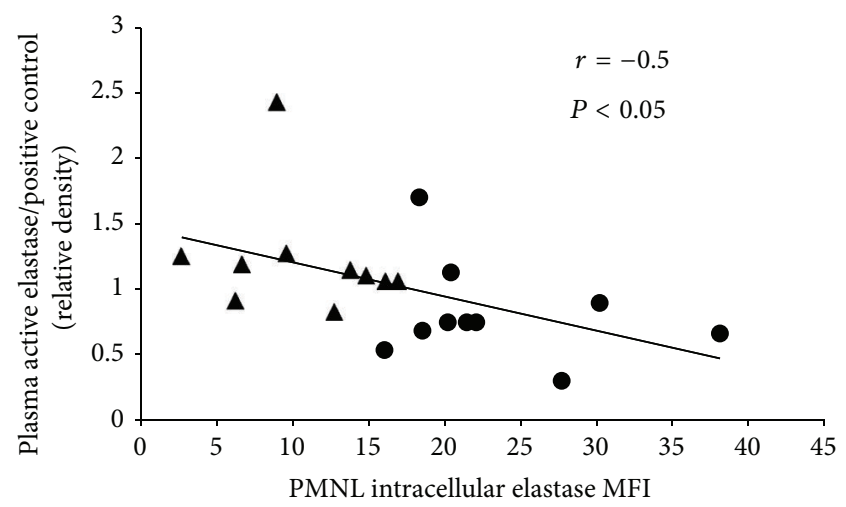

(d)

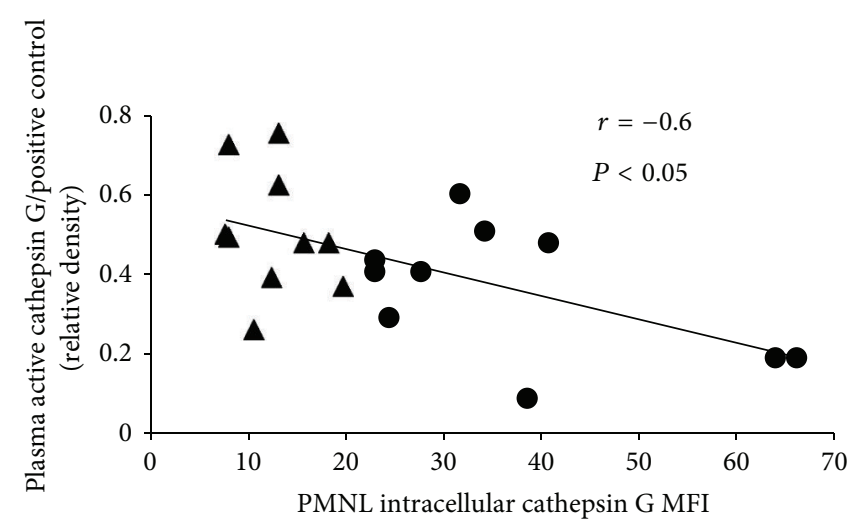

(e)

FIGURE 3: Levels of elastase and cathepsin G in HD and NC plasma. Proteins of plasma samples depleted from albumin and immunoglobulins of NC subjects and HD patient were separated on SDS-PAGE followed by western blot analysis. (a) A representative western blot of elastase and cathepsin G. Bands were visible at $30 \mathrm{kDa}$ (inhibitor unbound) and higher M.W complexes of 40, 50, and $70 \mathrm{kDa}$ in NC and HD plasma. ((b), (c)) Densities of unbound elastase and cathepsin $\mathrm{G}(30 \mathrm{kDa})$ from NC subjects and HD patients' plasma relative to commercial enzymes bands $\left({ }^{*} P>0.05\right.$ HD versus NC; $\left.n=10\right)$. (d) A negative correlation between plasma elastase levels of NC $(\bullet)$ and HD $(\boldsymbol{\Delta})$ and the expression of their PMNL membrane CD11b measured in whole blood $(r=-0.5 ; P<0.05)$. (e) A negative correlation between plasma cathepsin $\mathrm{G}$ levels of NC $(\bullet)$ and HD $(\boldsymbol{\Delta})$ and the expression of their PMNL membrane CD11b measured in whole blood $(r=-0.6 ; P<0.05)$. 


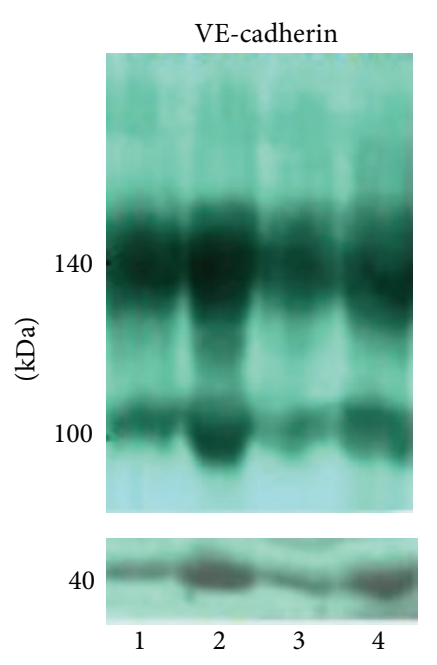

(a)

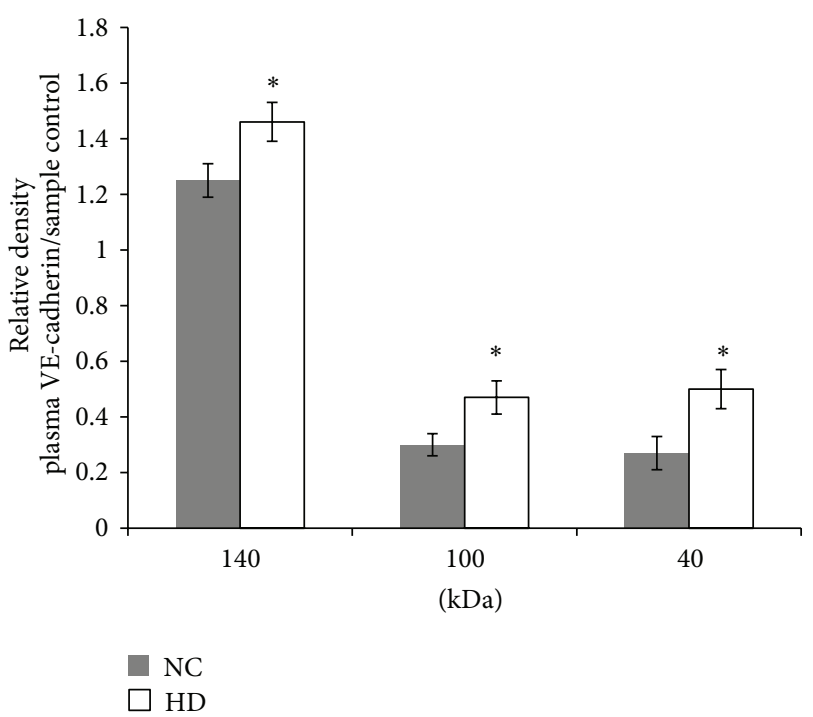

(b)

FIGURE 4: Soluble VE-cadherin fragments ( 140 and $100 \mathrm{kDa}$ forms) in HD plasma versus NC plasma.Proteins of plasma samples depleted from albumin and immunoglobulins of NC subjects and HD patient were separated on SDS-PAGE followed by western blot analysis (a) Representative gels showing three sizes of VE-cadherin: the whole molecule: $140 \mathrm{kDa}$ and the cleaved forms: $100 \mathrm{and} 40 \mathrm{kDa}$ in NC (1,3) and HD plasma $(2,4)$. (b) Relative densities of VE-cadherin from NC subjects and HD patient plasma $\left({ }^{*} P>0.05\right.$ HD versus NC; $\left.n=10\right)$.

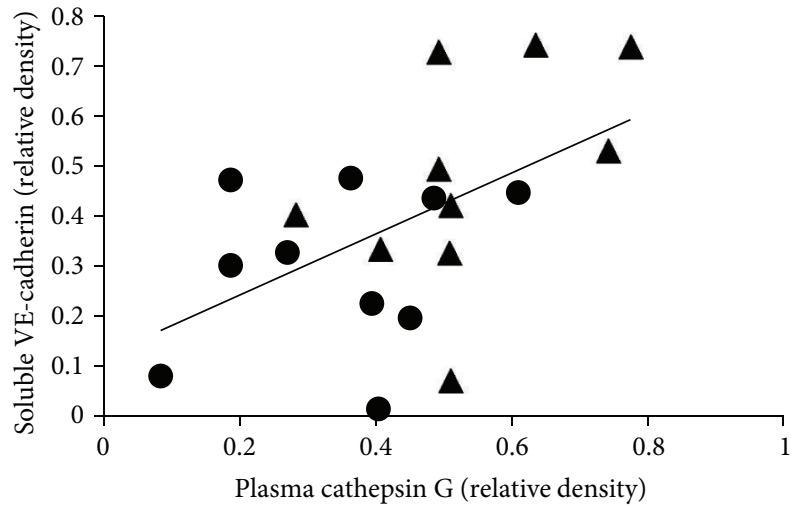

(a)

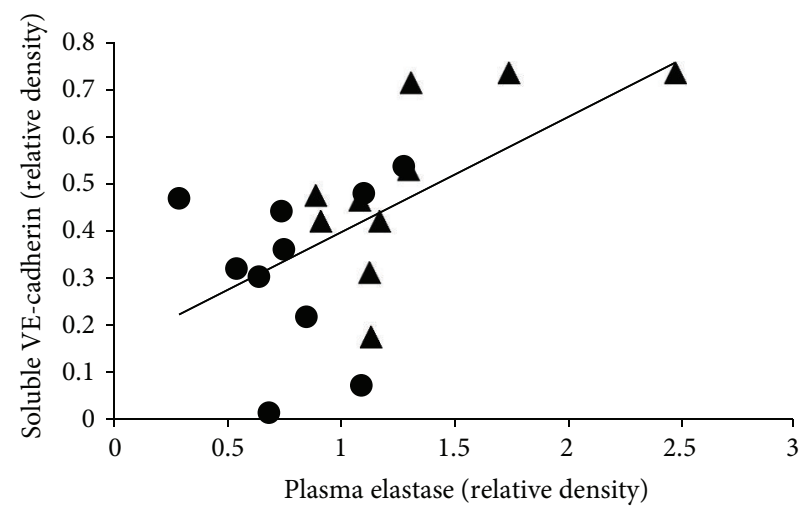

(b)

Figure 5: (a) Positive correlations between plasma active cathepsin G in plasma of NC ( $\bullet$ ) and HD patients $(\boldsymbol{\Delta})$ and soluble VE-cadherin $100 \mathrm{kDa}$ fragment $(r=0.51 ; P<0.05$; continuous line). (b) Positive correlations between plasma active elastase in plasma of NC and HD patients and VE-cadherin $100 \mathrm{kDa}$ fragment $(r=0.53 ; P<0.05$; continuous line).

This study demonstrates that HD plasma contains higher levels of soluble VE-cadherin present in three molecular forms: whole $140 \mathrm{kDa}$ molecule and two fragments, the 100 and the $40 \mathrm{kDa}$ forms. The $100 \mathrm{kDa}$ protein is probably a product of the proteolysis of VE-cadherin by elastase and cathepsin G [17]. This assumption is supported by the significant positive correlation found between the amount of plasma active elastase and cathepsin $\mathrm{G}$ and the plasma levels of the $100 \mathrm{kDa}$ fragment, the product of VE-cadherin degradation.

Our in vitro experiments showed that HD PMNLs can mediate cleavage of VE-cadherin from endothelial cells. This cleavage resulted in intense staining of the $40 \mathrm{kDa}$ fragment on endothelial cells concomitantly with the appearance of the $100 \mathrm{kDa}$ in the culture media. These results apparently conflict with our findings regarding low levels of elastase and cathepsin G found in HD PMNLs compared to controls. Yet, we propose that while in NC PMNLs these enzymes are stored within specific granules, HD PMNLs secrete their enzyme content to the surrounding milieu. Therefore, even trace amounts could elicit VE-cadherin cleavage as describe herein. The potential role of elastase and cathepsin $G$ released from primed HD PMNLs in mediating VE-cadherin cleavage is further supported by our in vitro experiments where applying purified elastase and cathepsin G to HUVEC resulted in an increase staining of the $40 \mathrm{kDa}$ fragment. 


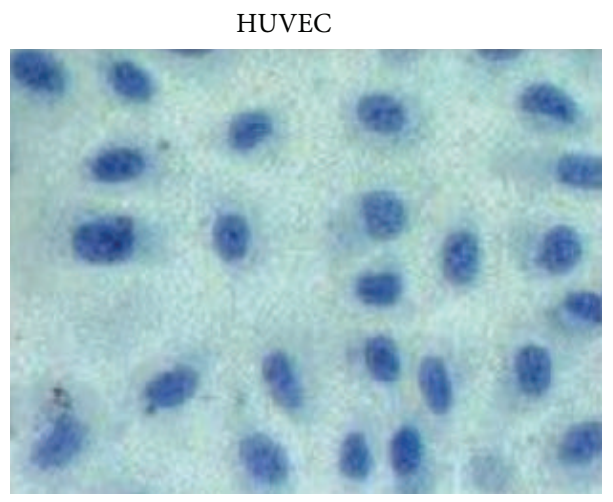

(a)

Cocultivation of HUVEC with NC PMNLs

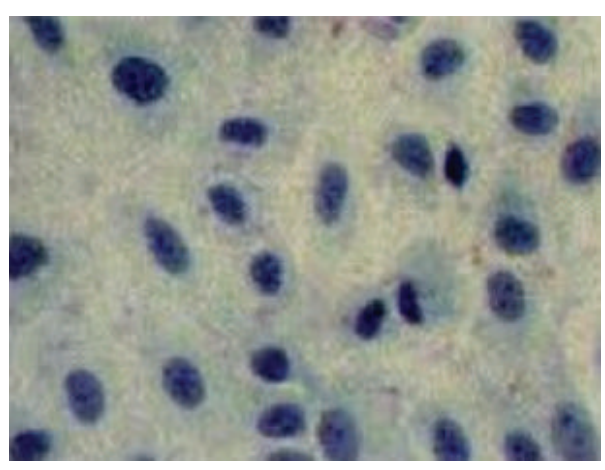

(c)

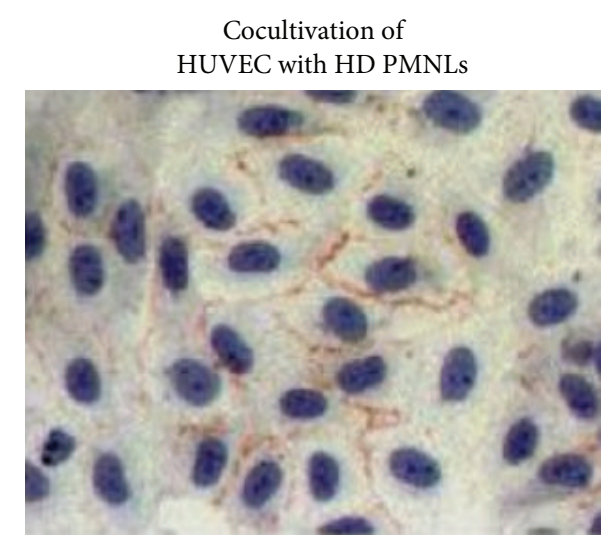

(b)

HUVEC + elastase and cathepsin $\mathrm{G}$

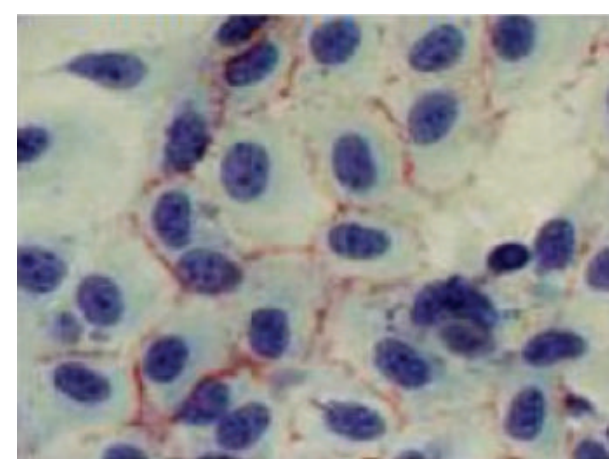

(d)

FIGURE 6: Indirect immunohistochemical staining of VE-cadherin ( $40 \mathrm{kDa}$ form) in HUVEC after cocultivation with PMNLs and after exposure to elastase and cathepsin G (light microscopy, magnification $\times 20$ ).

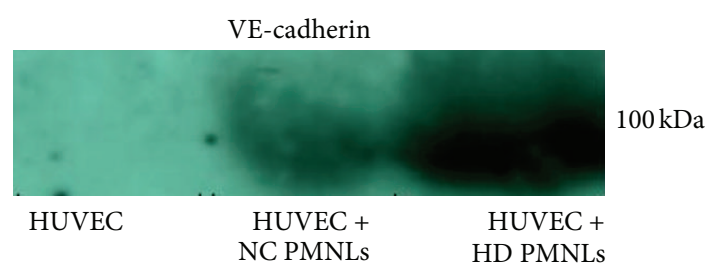

Figure 7: A representative gel showing soluble VE-cadherin ( $100 \mathrm{kDa}$ form) in PMNLs and HUVEC cocultivation media separated by SDS-PAGE followed by western blot analysis.

Our in vitro studies utilizing these proteinases on endothelial cells are in agreement with previous study demonstrating that after treatment with cathepsin G, endothelial cells became contracted and did not interact with neighbor cells. These effects depended on concentration and length of exposure to cathepsin G and ultimately cells became star-shaped until totally detached [26].

For conclusion, this study shows decreased elastase and cathepsin G expression in HD PMNLs while increased in HD plasma, most importantly in their active form. Furthermore, higher levels of soluble VE-cadherin were found in HD patient plasma. Thus, this study can provide a mechanism by which active elastase and cathepsin $G$ are released from primed PMNLs, mediate VE-cadherin cleavage, and initiate endothelial dysfunction in these patients. Moreover, the implications of this study are beyond HD patients and can be implicated in all clinical states associated with primed PMNLs and accelerated atherosclerosis such as hypertension and diabetes.

\section{Conflict of Interests}

The authors declare that there is no conflict of interests regarding the publication of this paper.

\section{Authors' Contribution}

Meital Cohen-Mazor and Rafi Mazor equally contributed to the preparation of this paper.

\section{Acknowledgments}

This research was supported in part by a Grant of the public committee for the designation of estate funds, the Ministry of Justice, Israel $(6014,3-1726)$. The authors are grateful to MSc. G. Shapiro for her excellent technical assistance in this project. 


\section{References}

[1] G. Feinstein and A. Janoff, "A rapid method for purification of human granulocyte cationic neutral proteases: purification and characterization of human granulocyte chymotrypsin like enzyme," Biochimica et Biophysica Acta, vol. 403, no. 2, pp. 477492, 1975.

[2] K. Ohlsson and I. Olsson, "The neutral proteases of human granulocytes. Isolation and partial characterization of granulocyte elastases," European Journal of Biochemistry, vol. 42, no. 2, pp. 519-527, 1974.

[3] A. Janoff, "Human granulocyte elastase. Further delineation of its role in connective tissue damage," American Journal of Pathology, vol. 68, no. 3, pp. 579-592, 1972.

[4] H. Keiser, R. A. Greenwald, G. Feinstein, and A. Janoff, "Degradation of cartilage proteoglycan by human leukocyte granule neutral proteases: a model of joint injury. II. Degradation of isolated bovine nasal cartilage proteoglycan," Journal of Clinical Investigation, vol. 57, no. 3, pp. 625-632, 1976.

[5] M. Ziff, H. J. Gribetz, and J. Lospalluto, "Effect of leukocyte and synovial membrane extracts on cartilage mucoprotein," Journal of Clinical Investigation, vol. 39, no. 2, pp. 405-412, 1960.

[6] A. Janoff and J. D. Zeligs, "Vascular injury and lysis of basement membrane in vitro by neutral protease of human leukocytes," Science, vol. 161, no. 3842, pp. 702-704, 1968.

[7] A. Heidland, W. H. Horl, and N. Heller, "Proteolytic enzymes and catabolism: enhanced release of granulocyte proteinases in uremic intoxication and during hemodialysis," Kidney International Supplement, vol. 24, no. 16, pp. S27-S36, 1983.

[8] D. Farley, G. Salvesen, and J. Travis, "Molecular cloning of human neutrophil elastase," Biological Chemistry Hoppe-Seyler, vol. 369, no. 5, pp. 3-7, 1988.

[9] G. Döring, "The role of neutrophil elastase in chronic inflammation," American Journal of Respiratory and Critical Care Medicine, vol. 150, no. 6, pp. S114-S117, 1994.

[10] T. Chandra, R. Stackhouse, V. J. Kidd, K. J. H. Robson, and S. L. C. Woo, "Sequence homology between human $\alpha 1-$ antichymotrypsin, $\alpha 1$-antitrypsin, and antithrombin III," Biochemistry, vol. 22, no. 22, pp. 5055-5061, 1983.

[11] R. A. Stockley, "Proteolytic enzymes, their inhibitors and lung diseases," Clinical Science, vol. 64, no. 2, pp. 119-126, 1983.

[12] A. Janoff, L. Raju, and R. Dearing, "Levels of elastase activity in bronchoalveolar lavage fluids of healthy smokers and nonsmokers," American Review of Respiratory Disease, vol. 127, no. 5, pp. 540-544, 1983.

[13] S. D. Swain, T. T. Rohn, and M. T. Quinn, "Neutrophil priming in host defense: role of oxidants as priming agents," Antioxidants and Redox Signaling, vol. 4, no. 1, pp. 69-83, 2002.

[14] B. Kristal, R. Shurtz-Swirski, J. Chezar et al., "Participation of peripheral polymorphonuclear leukocytes in the oxidative stress and inflammation in patients with essential hypertension," American Journal of Hypertension, vol. 11, no. 8, pp. 921928, 1998.

[15] R. Shurtz-Swirski, S. Sela, A. T. Herskovits et al., "Involvement of peripheral polymorphonuclear leukocytes in oxidative stress and inflammation in type 2 diabetic patients," Diabetes Care, vol. 24, no. 1, pp. 104-110, 2001.

[16] S. Sela, R. Shurtz-Swirski, M. Cohen-Mazor et al., "Primed peripheral polymorphonuclear leukocyte: a culprit underlying chronic low-grade inflammation and systemic oxidative stress in chronic kidney disease," Journal of the American Society of Nephrology, vol. 16, no. 8, pp. 2431-2438, 2005.

[17] B. Herman, S. Bibert, E. Concord et al., "Identification of proteases involved in the proteolysis of vascular endothelium cadherin during neutrophil transmigration," Journal of Biological Chemistry, vol. 278, no. 16, pp. 14002-14012, 2003.

[18] D. Gulino, E. Delachanal, E. Concord et al., "Alteration of endothelial cell monolayer integrity triggers resynthesis of vascular endothelium cadherin," Journal of Biological Chemistry, vol. 273, no. 45, pp. 29786-29793, 1998.

[19] R. M. Schaefer, N. Herfs, W. Ormanns, W. H. Horl, and A. Heidland, "Change of elastase and cathepsin G content in polymorphonuclear leukocytes during hemodialysis," Clinical Nephrology, vol. 29, no. 6, pp. 307-311, 1988.

[20] J. W. Yoon, M. V. Pahl, and N. D. Vaziri, "Spontaneous leukocyte activation and oxygen-free radical generation in end-stage renal disease," Kidney International, vol. 71, no. 2, pp. 167-172, 2007.

[21] M. S. Krug and S. L. Berger, "First-strand cDNA synthesis primed with oligo(dT)," Methods in Enzymology, vol. 152, pp. 316-325, 1987.

[22] R. K. Hirata, S.-T. Chen, and S. C. Weil, "Expression of granule protein mRNAs in acute promyelocytic leukemia," Hematologic Pathology, vol. 7, no. 4, pp. 225-238, 1993.

[23] E. A. Jaffe, R. L. Nachman, C. G. Becker, and C. R. Minick, "Culture of human endothelial cells derived from umbilical veins. Identification by morphologic and immunologic criteria," Journal of Clinical Investigation, vol. 52, no. 11, pp. 2745-2756, 1973.

[24] N. Lanir, M. Zilberman, I. Yron, G. Tennenbaum, Y. Shechter, and B. Brenner, "Reactivity patterns of antiphospholipid antibodies and endothelial cells: effect of antiendothelial antibodies on cell migration," Journal of Laboratory and Clinical Medicine, vol. 131, no. 6, pp. 548-556, 1998.

[25] J. Jacobi, S. Sela, H. I. Cohen, J. Chezar, and B. Kristal, "Priming of polymorphonuclear leukocytes: a culprit in the initiation of endothelial cell injury," American Journal of Physiology: Heart and Circulatory Physiology, vol. 290, no. 5, pp. H2051-H2058, 2006.

[26] L. Iacoviello, V. Kolpakov, L. Salvatore et al., "Human endothelial cell damage by neutrophil-derived cathepsin G: role of cytoskeleton rearrangement and matrix-bound plasminogen activator inhibitor-1," Arteriosclerosis, Thrombosis, and Vascular Biology, vol. 15, no. 11, pp. 2037-2046, 1995.

[27] N. Borregaard, K. Theilgaard-Mönch, O. E. Sørensen, and J. B. Cowland, "Regulation of human neutrophil granule protein expression," Current Opinion in Hematology, vol. 8, no. 1, pp. 23-27, 2001.

[28] J. B. Cowland and N. Borregaard, "The individual regulation of granule protein mRNA levels during neutrophil maturation explains the heterogeneity of neutrophil granules," Journal of Leukocyte Biology, vol. 66, no. 6, pp. 989-995, 1999.

[29] R. Ross, "The pathogenesis of atherosclerosis: a perspective for the 1990s," Nature, vol. 362, no. 6423, pp. 801-809, 1993.

[30] T. G. DeLoughery and S. H. Goodnight, "The hypercoagulable states: diagnosis and management," Seminars in Vascular Surgery, vol. 6, no. 1, pp. 66-74, 1993.

[31] T. Soeki, Y. Tamura, H. Shinohara, K. Sakabe, Y. Onose, and N. Fukuda, "Elevated concentration of soluble vascular endothelial cadherin is associated with coronary atherosclerosis," Circulation Journal, vol. 68, no. 1, pp. 1-5, 2004. 


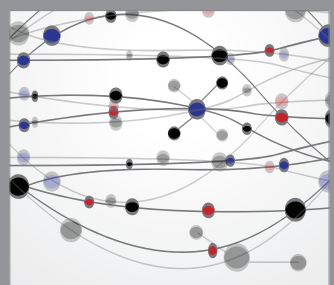

The Scientific World Journal
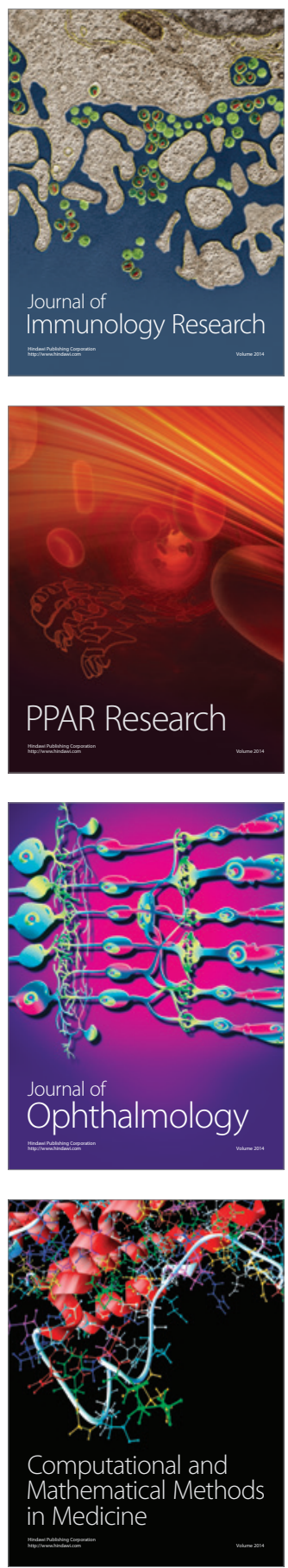

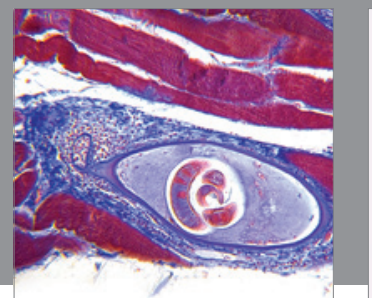

Gastroenterology

Research and Practice
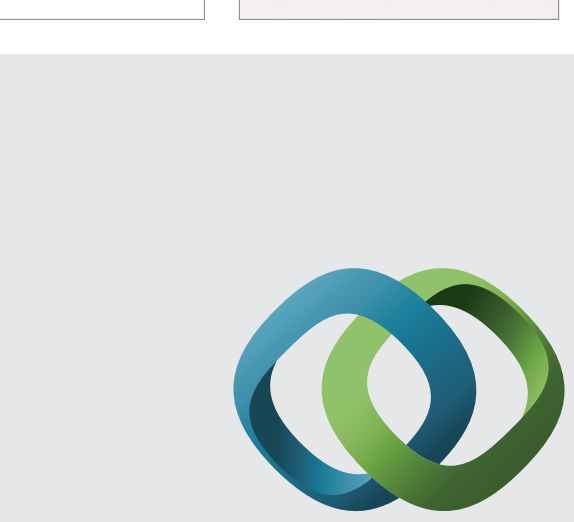

\section{Hindawi}

Submit your manuscripts at

http://www.hindawi.com
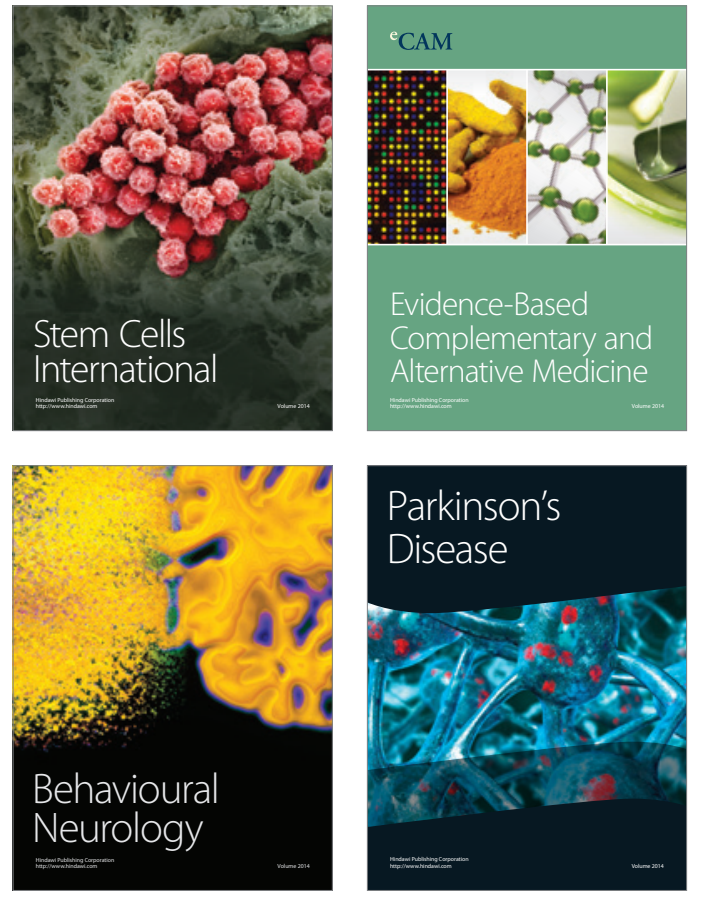
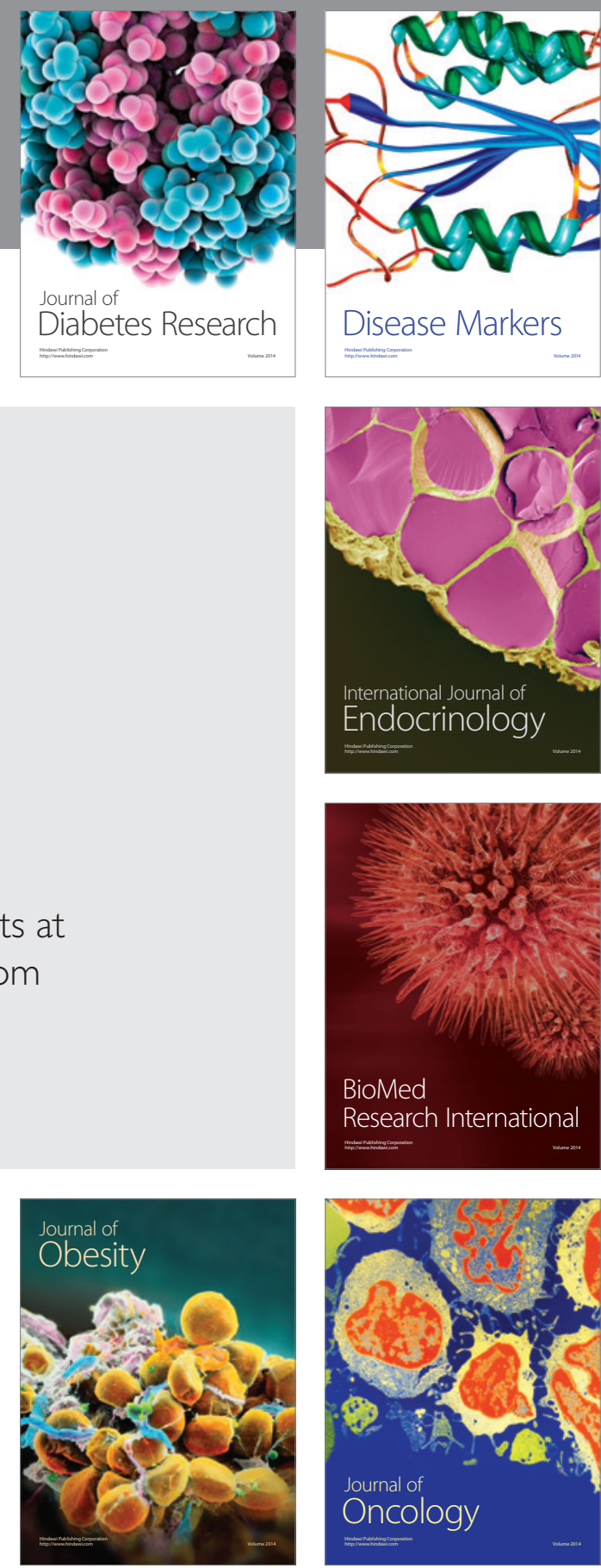

Disease Markers
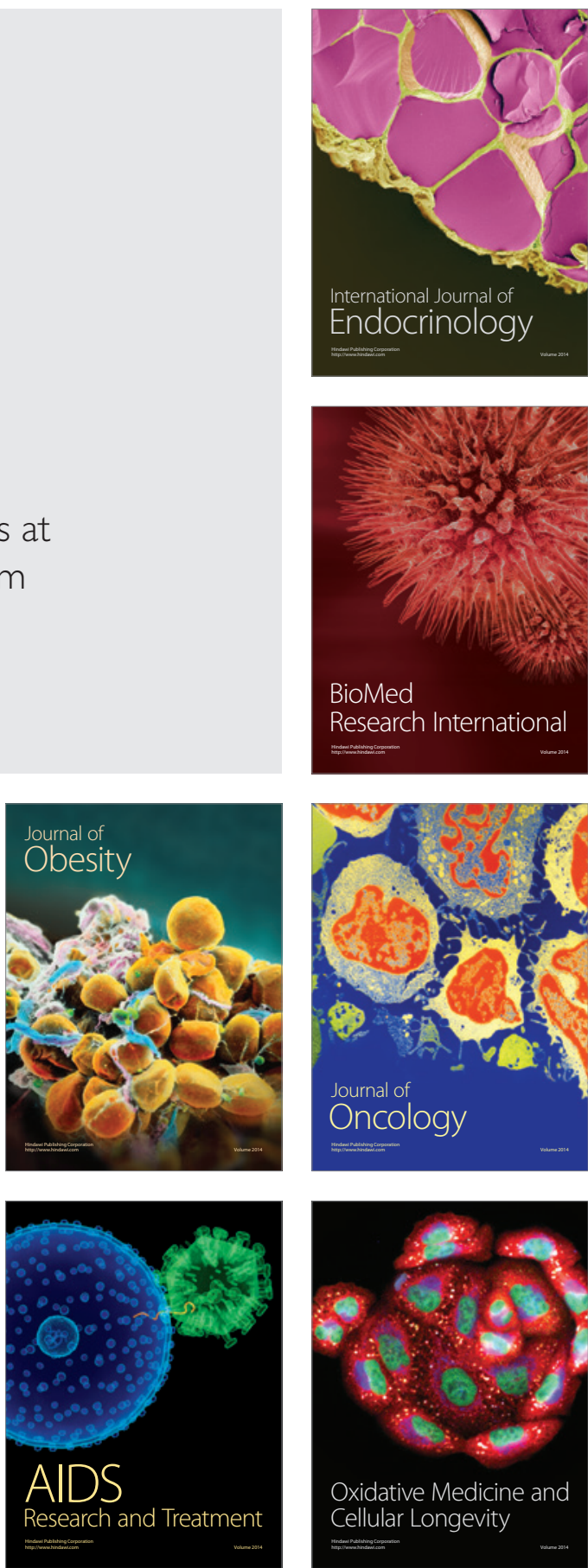